\title{
Übersichtsartikel
}

Andreas Reiner*

\section{Neurotransmittergesteuerte Rezeptoren in neuem Licht: Optische Methoden zur Kontrolle physiologischer Funktion}

https://doi.org/10.1515/nf-2017-0049

Zusammenfassung: Neurotransmittergesteuerte Rezeptoren sind auf vielfältige Weise an der synaptischen Signalleitung und Modulation beteiligt. Am Beispiel der Glutamatrezeptoren wird schnell deutlich, dass diese Rezeptorfamilien eine enorme Diversität aufweisen und es daher eine große Herausforderung bleibt, die unterschiedlichen Funktionen nah miteinander verwandter Rezeptorsubtypen aufzuklären. Pharmakologische und genetische Methoden werden zunehmend durch optogenetische Ansätze ergänzt, welche es erlauben, das Rezeptorsignaling mit Licht zu manipulieren. Am Beispiel der Glutamatrezeptoren lege ich dar, wie kovalent verankerte photoschaltbare Liganden genutzt werden können, um einzelne Rezeptorsubtypen mit hoher räumlicher und zeitlicher Präzision in ausgewählten Zellen zu kontrollieren. Mit diesen und ähnlichen Techniken eröffnen sich neue, vielversprechende Möglichkeiten, die Funktion bestimmter Rezeptoren im Nervensystem zu untersuchen.

Schlüsselwörter: G-Protein-gekoppelte Rezeptoren; Glutamatrezeptoren; ligandengesteuerte Ionenkanäle; Optogenetik; Optopharmakologie

\section{Einleitung}

Unser Nervensystem ist ein ausgesprochen komplexes und leistungsstarkes Organ. Seine Leistungsfähigkeit ist auf eine Vielzahl verschiedener molekularer Mechanismen, Zelltypen und Verbindungen zwischen spezialisierten Hirnregionen zurückzuführen. Diese Diversität zu erfassen und zu katalogisieren, dürfte ein wichtiger Schlüssel für das Verständnis physiologischer und pathophysiologischer Hirnfunktionen sein.

*Korrespondenzautor: Andreas Reiner, Fakultät für Biologie und Biotechnologie, Ruhr-Universität Bochum, Bochum, Germany, E-Mail: andreas.reiner@rub.de
Aktuelle experimentelle Ansätze zielen darauf ab, dieser Komplexität auf allen Organisationsebenen gerecht $\mathrm{zu}$ werden, beispielsweise indem man versucht, die verschiedenen Zelltypen und Verbindungen mithilfe genetischer Methoden genauer zu kartieren. Ein wichtiger Forschungsgegenstand bleiben auch die verschieden Formen der Signalprozessierung innerhalb einzelner Neurone und Synapsen. Zu diesem Zweck wäre es nicht nur hilfreich, die Transmission und Integration von Signalen auf der Ebene einzelner Synapsen messen und visualisieren $\mathrm{zu}$ können, d.h. im Größenbereich einzelner Dornenfortsätze $(<1 \mu \mathrm{m})$ und auf schnellen Zeitskalen (Millisekunden), sondern auch, diese Prozesse mit hoher Präzision manipulieren zu können. In diesem Übersichtsartikel beschreibe ich, welche Herausforderungen mit der Untersuchung der an der synaptischen Transmission und Modulation beteiligten Rezeptoren einhergehen und erläutere, wie neue, lichtkontrollierte Werkzeuge genutzt werden können, um die Funktion einzelner Rezeptorsubtypen besser zu verstehen. Den Schwerpunkt lege ich auf lichtgesteuerte Glutamatrezeptoren (GluRs), die Fragestellungen und methodischen Ansätze sind jedoch allgemein auf neurotransmittergesteuerte Rezeptoren anwendbar.

\section{Herausforderungen und klassische Methoden für die Funktionsunter- suchung neurotransmitter- gesteuerter Rezeptoren}

Die molekularen Ereignisse, die der synaptischen Transmission, Integration und Plastizität zugrunde liegen, werden seit geraumer Zeit untersucht. Die Wirkung der verschiedenen chemischen Neurotransmitter wurde früh erkannt, und mittels intensiver Klonierungsstudien konnten die Rezeptoren identifiziert werden, die diese Signale dekodieren. Die meisten Neurotransmitter werden von zwei Rezeptorklassen detektiert: erstens, ionotropen Rezeptoren, bei 
A
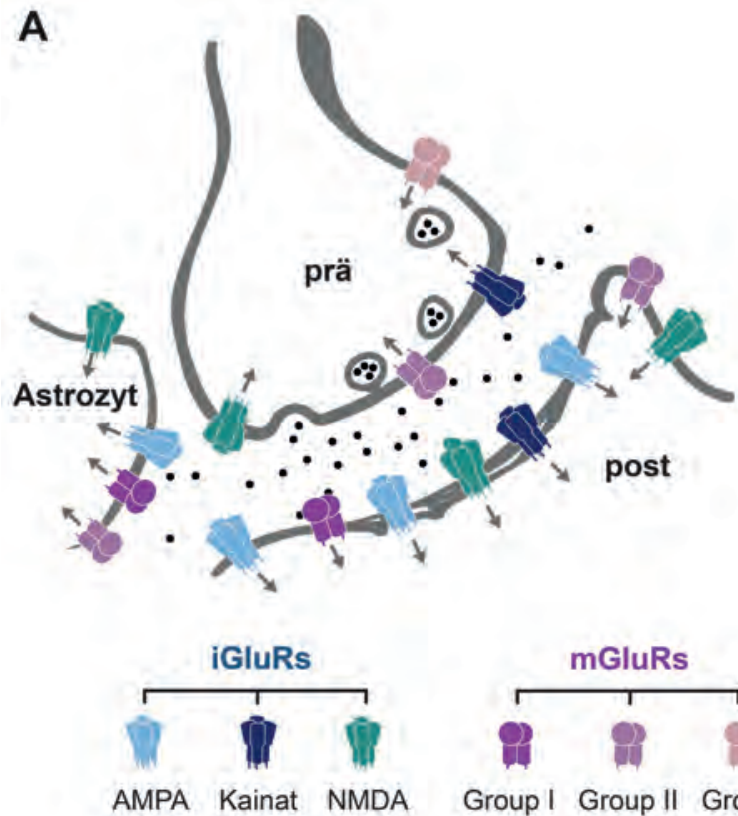

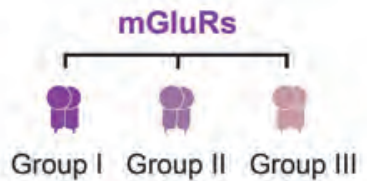

B

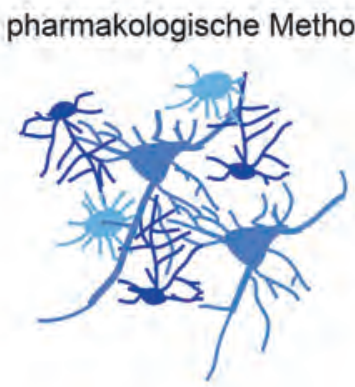

genetische Methoden

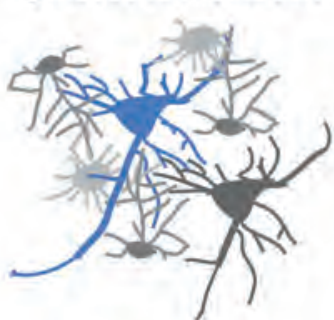

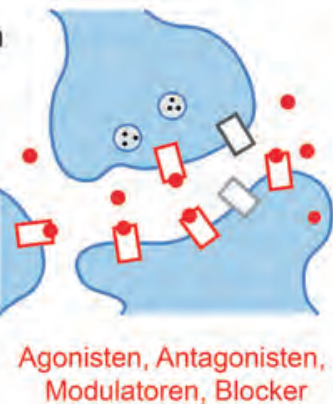

Modulatoren, Blocker

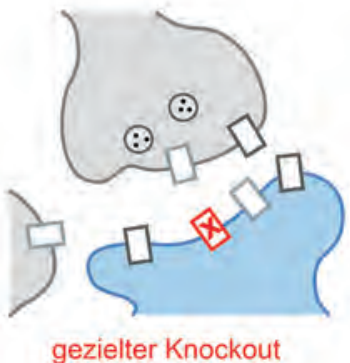

Abb. 1: Neurotransmittergesteuerte Rezeptoren am Beispiel einer glutamatergen Synapse und experimentelle Untersuchungsmöglichkeiten. A: Verschiedene ionotrope und metabotrope Glutamatrezeptorsubtypen, iGluRs (blau/grün) und mGluRs (violet), sind postsynaptisch, aber auch präsynaptisch und auf Gliazellen, hier einem Astrozyten, lokalisiert. Die Funktionen der einzelnen GluRs, die an der synaptischen Transmission, Homöostase und Plastizität beteiligt sind, sind daher nur schwer voneinander abzugrenzen. B: Pharmakologische Manipulationen können zwischen einem Teil der verschiedenen Rezeptorsubtypen unterscheiden, aber nicht zwischen Rezeptoren in verschiedenen Zellen und synaptischen Kompartimenten. C: Genetische Methoden, wie z. B. der Knockout von Rezeptoren, bieten hingegen höchstmögliche Spezifität, aber nur eine geringe Zeitauflösung.

denen es sich um ligandengesteuerte Ionenkanäle handelt, die einen Stromfluss vermitteln, und zweitens, metabotropen Rezeptoren, die zur Klasse der G-Protein-gekoppelten Rezeptoren (GPCRs) gehören, welche über die Aktivierung von G-Proteinen Sekundärbotenstoffe kontrollieren. Ein typisches Beispiel ist Glutamat, der wichtigste exzitatorische Neurotransmitter und ein wichtiger Neuromodulator im zentralen Nervensystem von Wirbeltieren, das von ionotropen GluRs (iGluRs) und metabotropen GluRs (mGluRs) detektiert wird (Abbildung 1A). Beide Rezeptorklassen umfassen eine Vielzahl verschiedener Subtypen und Isoformen mit unterschiedlichen Eigenschaften (Monyer et al., 1991; Hollmann und Heinemann, 1994; Traynelis et al., 2010; Reiner und Levitz, 2018). Ihre funktionelle Diversität wird durch die Bildung von heteromeren Rezeptoren und die Assoziation mit akzessorischen Untereinheiten und Gerüstproteinen noch erhöht (Schwenk et al., 2012; Jacobi und von Engelhardt, 2017).

Die physiologischen Funktionen der einzelnen iGluRund mGluR-Subtypen zu bestimmen, bleibt eine große Herausforderung. Viele Rezeptorsubtypen sind im Nervensystem weit verbreitet und die meisten Neurone exprimieren verschiedene Rezeptoruntereinheiten, die sich auch innerhalb einzelner Zellen unterschiedlich lokalisieren. So treten beispielsweise iGluRs, die dafür bekannt sind, erregende postsynaptische Depolarisationen zu erzeugen, auch in präsynaptischen Endungen und in Gliazellen auf, was auf zusätzliche Funktionen im Bereich der Neuromodulation und Gliotransmission hindeutet (Reiner und Levitz, 2018). Komplex wird die Situation auch dadurch, dass die Rezeptorpopulationen und deren Zusammensetzung an den einzelnen Synapsen nicht statisch, sondern variabel ist. Diese Anpassungsmechanismen ermöglichen die Integration verschiedener Signale und bilden somit die Grundlage für die synaptische Homöostase und Plastizität. Eine solche Komplexität ist nicht nur bei GluRs, sondern auch bei den meisten anderen neurotransmittergesteuerten Rezeptoren zu finden. Zusammenfassend kann festgestellt werden, dass wir oftmals keine genaue Kenntnis darüber haben, wie die einzelnen Subtypen einer Rezeptorfamilie zu den verschiedenen physiologischen Funktionen beitragen.

Für die funktionelle Analyse von neurotransmittergesteuerten Rezeptoren stehen im Wesentlichen pharmakologische und genetische Methoden zur Verfügung (Abbildung $1 \mathrm{~B}, \mathrm{C}$ ). Pharmakologische Wirkstoffe, also Agonisten, Antagonisten, allosterische Modulatoren und Blocker, erlauben es, die Rezeptoraktivierung zu beeinflussen und somit auf die physiologische Funktion zu schlie- 
ßen. So wurden beispielsweise die iGluR-Agonisten AMPA ( $\alpha$-Amino-3-hydroxy-5-methyl-4-isoxazolpropionsäure), Kainat und NMDA ( $N$-Methyl-D-Aspartat) genutzt, um die entsprechenden iGluR-Unterfamilien $\mathrm{zu}$ unterscheiden und einzuteilen. Trotz intensiver Bemühungen, Substanzen mit hoher Selektivität für einzelne Rezeptorsubtypen zu entwickeln, ist es jedoch weiterhin nicht möglich, die verschiedenen iGluR-Subtypen getrennt voneinander $\mathrm{zu}$ aktivieren oder zu inhibieren (Traynelis et al., 2010). Ferner kann durch die Applikation pharmakologischer Substanzen nicht zwischen Rezeptoren in verschiedenen Zelltypen oder in verschiedenen synaptischen Kompartimenten unterschieden werden (Abbildung 1B), um z.B. prä- von postsynaptischen Effekten zu trennen.

Genetische Manipulationen stellen einen komplementären Ansatz dar, um die Rolle neurotransmittergesteuerter Rezeptoren zu untersuchen. Sie erlauben es, die Expression einzelner Rezeptoruntereinheiten zu unterbinden, zu verringern oder zu erhöhen, oder modifizierte Rezeptoruntereinheiten einzuführen (Luo et al., 2018). Die genetische Manipulation beschränkt sich auf die jeweilige Rezeptoruntereinheit und kann z. B. durch Verwendung von bestimmen Treiberlinien, Promotoren oder stereotaktischen Injektionen auch auf ausgewählte Zellen oder Hirnareale beschränkt werden (Abbildung 1C). Transgene Tiere und virusbasierte Werkzeuge, die heute in großer Zahl zur Verfügung stehen, machen diesen Ansatz besonders attraktiv. Nachteilig ist, dass genetische Manipulationen oftmals relativ langsam sind, und damit auf der Zeitskala vieler physiologischer Prozesse nicht an- und abgeschaltet werden können.

\section{Lichtbasierte Methoden und die Entwicklung der Optogenetik}

Lichtbasierte Methoden bieten für die Untersuchung biologischer Systeme eine Reihe an Vorteilen. Licht im sichtbaren Bereich interferiert nur geringfügig mit den meisten
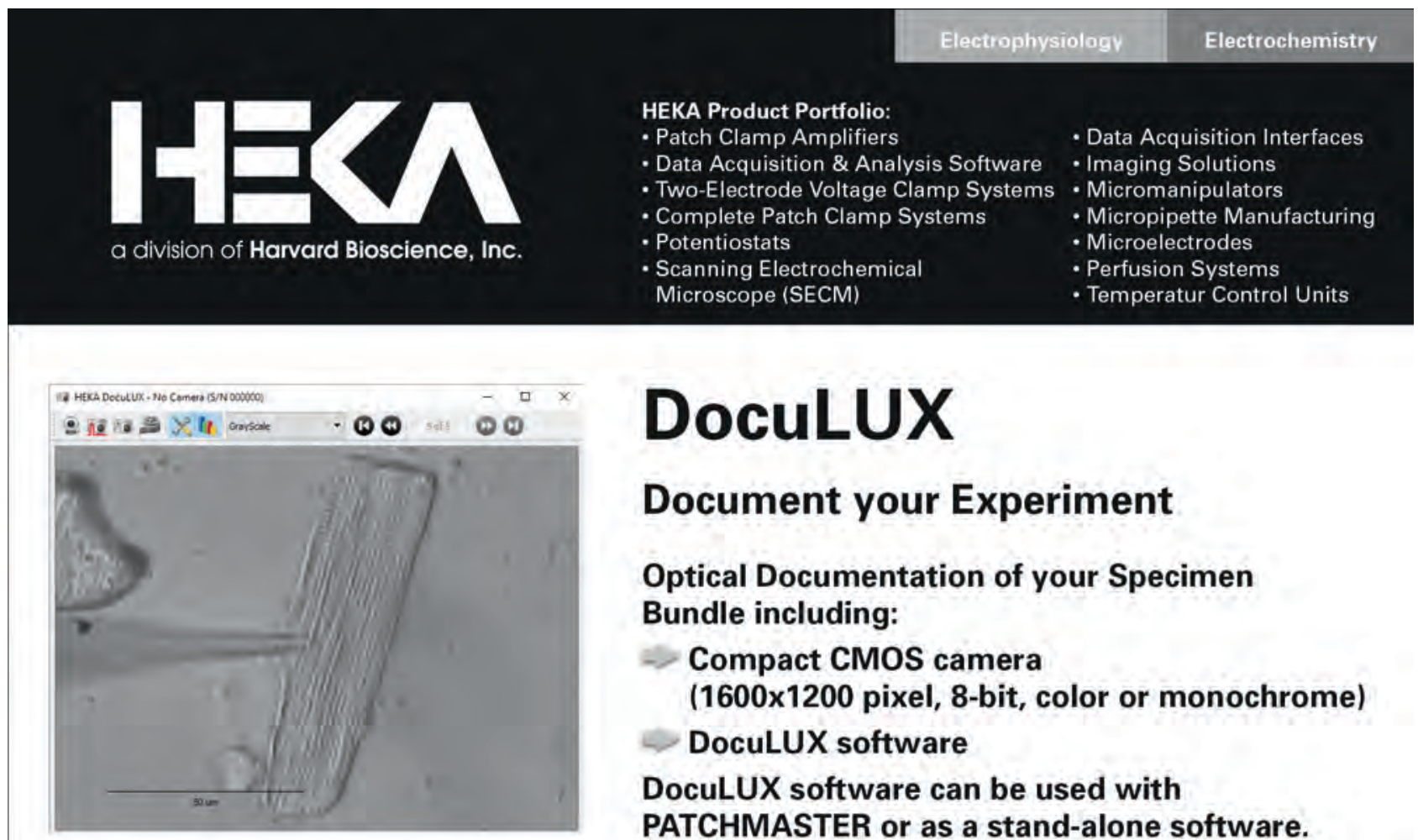

\title{
DocuLUX
}

\section{Document your Experiment}

\author{
Optical Documentation of your Specimen \\ Bundle including: \\ Compact CMOS camera \\ (1600x1200 pixel, 8-bit, color or monochrome) \\ DocuLUX software \\ DocuLUX software can be used with \\ PATCHMASTER or as a stand-alone software.
}


biologischen Prozessen und ist daher wenig invasiv. Rotes Licht und Licht im nahen Infrarotbereich kann dabei sogar tief in Gewebe eindringen. Ein vielleicht noch wichtigerer Vorzug lichtbasierter Methoden ist die hohe räumliche und zeitliche Auflösung, die im Vergleich zu pharmakologischen und genetischen Methoden erzielt werden kann. Diese Vorteile werden auch mit der umfassenden Anwendung fluoreszenzbasierter Imagingverfahren deutlich, welche heute $u$.a. in vivo genutzt werden, mittels ZweiPhotonen-Anregung eine hohe Auflösung in Geweben bieten, oder, im Falle super-auflösender Verfahren, Details im Bereich von zehn Nanometern auflösen können.

Licht erscheint daher nicht nur als ein ideales Werkzeug für die Beobachtung, sondern auch für die Manipulation biologischer Prozesse. Eine häufig genutzte Technik ist das Photouncaging (Ellis-Davies, 2007), das Mitte der 1980er zunächst auf Sekundärbotenstoffe und kurze Zeit später von George Hess auf Acetylcholin und Glutamat angewendet wurde. Die biologisch aktive Substanz wird dabei mit einer photospaltbaren Schutzgruppe modifiziert (maskiert), in dieser inaktiven Form zum biologischen Präparat gegeben, und bei Bedarf mithilfe von Licht freigesetzt. Diese Methode ist mithilfe von Laserlichtquellen experimentell leicht $\mathrm{zu}$ implementieren und erlaubt es, Substanzen innerhalb von Millisekunden und im Bereich von Mikrometern freizusetzen. Für alle wichtigen neurotransmittergesteuerten Rezeptoren stehen gecagte Agonisten zur Verfügung, und zum Teil wurden auch gecagte Blocker und Neuropeptide entwickelt, oftmals mit unterschiedlichen spektralen Eigenschaften, z. B. um eine effiziente Zwei-Photonen-Anregung zu ermöglichen (EllisDavies, 2007; Reiner et al., 2015). Da das Photouncaging einen irreversiblen Schritt darstellt, bestimmt die Diffusion (oder die Aufnahme) der freigesetzten Substanz, wie schnell das Signal abklingt.

Eine Alternative zum Photouncaging von Neurotransmittern ist die Verwendung photoschaltbarer Analoga. Hierbei wird der gewünschte Ligand mit einer photoschaltbaren Gruppe modifiziert, zumeist einem Azobenzol. Azobenzole zeigen eine reversible, lichtinduzierte trans-cis-Isomerisierung, die in einer erheblichen Konformationsänderung des Liganden resultiert, und damit die Bindungsaffinität für den Zielrezeptor verändert. Licht bestimmter Wellenlängen kann somit genutzt werden, um den Liganden zwischen hoch- und niedrigaffinen Konfigurationen zu schalten. Photoschaltbare Agonisten, Antagonisten und Modulatoren wurden für eine Vielzahl an Rezeptoren entwickelt, so auch für iGluRs und mGluRs (Pittolo et al., 2014; Laprell et al., 2015; Barber et al., 2017).

Photouncaging und photoschaltbare Liganden bieten für die meisten Anwendungen eine ausreichend hohe zeitliche Auflösung im Bereich weniger Millisekunden. Die räumliche Auflösung wird nur durch die Diffusion des Liganden beschränkt. Die Wirkung der Liganden kann jedoch nicht auf spezifische Zellen beschränkt werden, wodurch es beispielsweise nicht möglich ist, zwischen prä- und postsynaptischen Effekten zu unterschieden. Nichtsdestoweniger werden gecagte und photoschaltbare Liganden gerne genutzt, um endogene Rezeptorpopulationen mit Licht zu aktivieren oder neuronale Schaltkreise funktionell zu kartieren.

Ein wichtiger konzeptioneller Schritt kam mit der Idee, genetische Methoden zu nutzen, um optische Kontrollwerkzeuge in ausgewählten Zellen zu exprimieren (Zemelman und Miesenböck, 2001), ein Konzept, das als Optogenetik bekannt geworden ist (Deisseroth und Hegemann, 2017; Kim et al., 2017; Reiner und Isacoff, 2013). Eine frühe Umsetzung dieses Konzeptes wurde durch Photouncgaing von ATP oder Capsaicin in Kombination mit der heterologen Expression von ATP-gesteuerten P2X-Rezeptoren oder Capsaicin-sensitiven TRP-Kanälen erzielt (Zemelman et al., 2003). Kurze Zeit später wurden Opsine als ideale optogenetische Werkzeuge identifiziert, insbesondere, um die Aktivität von Neuronen zu kontrollieren (Boyden et al., 2005; Li et al., 2005; Nagel et al., 2005). Die Familie der Opsinproteine umfasst lichtgesteuerte GPCRs, z. B. Rhodopsine aus Vertebraten, lichtgetriebene Pumpen (Transporter) mikrobiellen Ursprungs, wie z. B. Halorhodopsin und Bakteriorhodopsin, sowie Kanalrhodopsine (channelrhodopsines), bei denen es sich um lichtgesteuerte Ionenkanäle handelt, die erstmals 2002 in Grünalgen identifiziert werden konnten (Nagel et al., 2002). Die Aktivierung wird in allen Fällen durch eine lichtinduzierte cis-trans- bzw. trans-cis-Isomerisierung des Retinalcofaktors vermittelt.

Viele Opsine werden in Neuronen gut exprimiert und können daher mit den vielfältigen genetischen stateof-the-art Methoden kombiniert werden. Ein besonders häufig verwendetes Werkzeug ist Kanalrhodopsin, das sich gut eignet, um ausgewählte Neurone mit Blaulichtpulsen zu depolarisieren (aktivieren), und daher in einer großen Breite an experimentellen Systemen zum Einsatz kommt, Experimente im lebenden Tier eingeschlossen (Kim et al., 2017). Kanalrhodopsin und andere Opsine wurden zudem gezielt modifiziert, um deren kinetische und spektrale Eigenschaften zu optimieren, oder deren Ionenselektivität zu verändern (Deisseroth und Hegemann, 2017; Wietek et al., 2017). Mit diesen Werkzeugen ist die lichtinduzierte Stimulation von Neuronen, und im geringeren Maße auch die lichtinduzierte Inhibition (Wiegert et al., 2017), in vielen Teilgebieten der Neurowissenschaften zu einer Standardmethode geworden. 
A

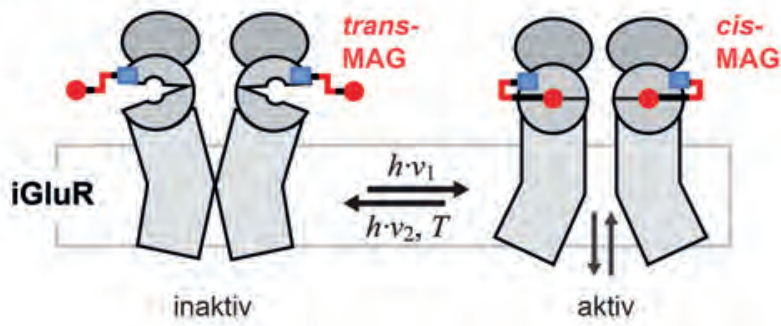

B

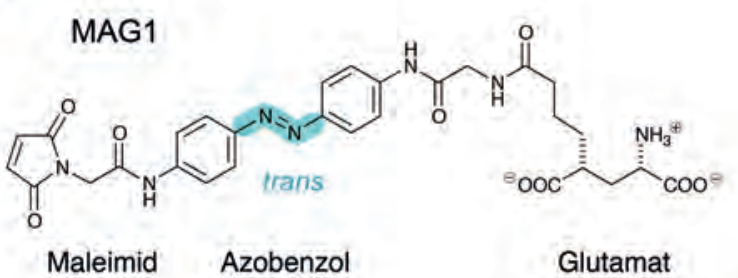

C

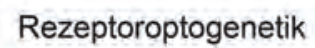

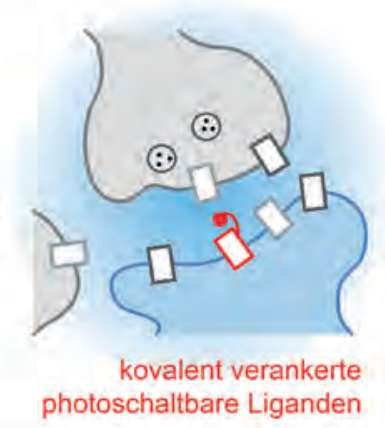

D GluK2(L439C)-MAGO

Abb. 2: Kovalent verankerte photoschaltbare Liganden ermöglichen es, Glutamatrezeptoren mit Licht zu kontrollieren. A: Schema, das die kovalente Kopplung photoschaltbarer Liganden (rot) an genetisch modifizierte Rezeptoruntereinheiten zeigt, die einen Cysteinrest (blau) in der Nähe der Ligandenbindetasche tragen. Durch Bestrahlung mit Licht kann der kovalent verankerte Ligand reversibel isomerisiert werden und somit den Rezeptor aktivieren (Photoagonismus). B: Chemische Struktur eines typischen MAG-Liganden (L-MAG1) der mit 375 nm bzw. $\sim 488 \mathrm{~nm}$ Licht isomerisiert werden kann. Andere MAG-Photoschalter reagieren auf andere Wellenlängen oder zeigen ein schnelle, spontane cis-zu-trans Umwandlung. C: Aufgrund der genetischen Kodierung der Anknüpfungsstelle ist die Manipulation selektiv für den modifizierten Rezeptorsubtyp und kann auf ausgewählte Zellpopulationen beschränkt werden. D: Beispiel für die Photoaktivierung von iGluRs (voltageclamp Ableitungen). Links, reversibles An- und Abschalten von heterolog exprimierten GluK2(L439C) Kainatrezeptoren, die mit einem MAG0-Photoschalter modifiziert wurden. Bestrahlung mit $380 \mathrm{~nm}$ Licht (violette Balken) führt zu Photoagonismus (Einwärtsströme), der mit $500 \mathrm{~nm}$ Licht (grüne Balken) wieder abgeschalten werden kann. Aufnahme in Gegenwart eines Desensitisierungsblockers. Rechts, schnelles Photoschalten mit kurzen Lichtpulsen hoher Intensität (Pfeile) führt zu Aktivierungs- und Desensitisierungskinetiken im Submillisekundenbzw. Millisekundenbereich. Teilabbildung D wurde modifiziert aus Reiner und Isacoff, 2014a entnommen.

\section{Optogenetische Kontrolle von neu- rotransmittergesteuerten Rezepto- ren mithilfe kovalent verankerter photoschaltbarer Liganden}

Opsine sind ideale Werkzeuge, um die neuronale Erregbarkeit zu modulieren. Parallel dazu wurden chemischoptogenetische Ansätze entwickelt, die es erlauben, auch Proteine, die natürlicherweise im Nervensystem vorkommen, mit Licht zu kontrollieren (Banghart et al., 2004; Volgraf et al., 2006). Ein solcher Ansatz ist die Verwendung von photoschaltbaren Liganden, die kovalent an genetisch modifizierte Rezeptoruntereinheiten gebunden werden (Abbildung 2). Dieser Ansatz kann wahlweise für die Photoaktivierung oder die Photoinhibition von iGluRs und mGluRs verwendet werden (Reiner et al., 2015), und ist auch auf andere Rezeptorfamilien anwendbar (Fehrentz et al., 2011; Kramer et al., 2013).
Die synthetischen, photoschaltbaren Liganden werden kovalent an den ausgewählten Rezeptoren verankert (Abbildung 2). Sie umfassen eine funktionelle Gruppe, die die Kopplung an eine definierte Position auf der Rezeptoroberfläche ermöglicht, eine photoschaltbare Gruppe, die bei Bestrahlung mit Licht ihre Konformation ändert, und eine Kopfgruppe, die als Ligand dient. Für die optische Aktivierung von GluRs können beispielsweise Maleimid-Azobenzol-Glutamat- (MAG) Photoschalter genutzt werden (Abbildung 2 A, B) (Volgraf et al., 2006; Gorostiza et al., 2007; Levitz et al., 2013). Die Maleimidgruppe erlaubt es, den MAG-Photoschalter kovalent an eine genetisch kodierte Cysteinseitenkette in der Nähe der Bindungstasche $\mathrm{zu}$ koppeln. Azobenzol fungiert als photoschaltbare Gruppe, die mit Licht verschiedener Wellenlängen zwischen der trans- und cis-Konfiguration geschalten werden kann, und die Glutamatkopfgruppe, im Fall von iGluRs ein 4-Alkylderivat in L-Konfiguration (D für mGluRs), dient als hocheffizienter Agonist. 
Ein entscheidender Vorteil dieses Ansatzes ist, dass die Anknüpfungsstelle genetisch kodiert ist. Auf diese Weise wird unmittelbar Spezifität für den gewählten Rezeptorsubtyp erreicht, da nur Rezeptoren mit einer MAGmodifizierten Untereinheit durch Licht angesprochen werden. Ferner wird es dadurch möglich, die Manipulation auf bestimmte Zelltypen und synaptische Kompartimente $\mathrm{zu}$ beschränken (Abbildung $2 \mathrm{C}$ ). Die räumliche Auflösung wird nur durch die Fokussierung des Lichtes auf die gewünschte Region beschränkt. Die Zeitauflösung wird im Wesentlichen durch die verwendete Lichtintensität bestimmt, wobei mit kurzen, intensiven Laserpulsen die Ligandenbindung und Dissoziation im Millisekundenbereich kontrolliert werden kann (Abbildung 2D) (Reiner und Isacoff, 2014a). Mit dieser Geschwindigkeit wird es möglich, synaptische Aktivierungen präzise nachzubilden, sie kann aber auch benutzt werden, um neue Informationen über den Schaltmechanismus von iGluRs und mGluRs zu erhalten (Reiner und Isacoff, 2014a; Levitz et al., 2016a).

Lichtgesteuerte GluRs, wie z.B. photoaktivierbare Varianten des Kainatrezeptors GluK2, wurden in einer Vielzahl von experimentellen Systemen erfolgreich eingesetzt. Diese umfassen kultivierte Zellen (Reiner und Isacoff, 2014b), primäre Neuronen- (Szobota et al., 2007) und Astrozytenkulturen (Li et al., 2012), Hirnschnitte (Berlin et al., 2016), Studien an der Retina ex vivo und in vivo (Gaub et al., 2014), an Fliegen (Kauwe et al., 2013), lebenden Zebrafischen (Wyart et al., 2009) und in Mäusen (Levitz et al., 2016b). In allen Fällen wurden keine Nebenwirkungen beobachtet und die Photoaktivierung blieb über lange Zeiträume robust und reversibel. Die erste Generation an MAG-Photoschaltern kann effizient mit $375 \mathrm{~nm}$ und $488 \mathrm{~nm}$ Licht kontrolliert werden. Durch Modifikation des Azobenzolschalters konnten aber auch MAG-Varianten erzeugt werden, die auf einzelne Blaulichtpulse $\left(\mathrm{MAG}_{460}\right)$ (Kienzler et al., 2013) oder auf rotes Licht (Rullo et al., 2014) ansprechen. Einige MAG-Varianten sind auch für Zwei-Photon-Anregung geeignet (Carroll et al., 2015). Ein Überblick über die derzeit verfügbaren MAG-Photoschalter für iGluRs ist in Levitz et al., 2016b zu finden.

MAG-Photoschalter wurden an einer Reihe verschiedener iGluR und mGluR Untereinheiten eingesetzt. Ein aktuelles Beispiel ist die optische Kontrolle verschiedener NMDA-Rezeptoruntereinheiten, die, neben anderen Funktionen, postsynaptische $\mathrm{Ca}^{2+}$-Signale erzeugen und die Plastizität von Synapsen kontrollieren. Mehrere MAG-Anknüpfungspositionen wurden etabliert, um einerseits Photoagonismus (Aktivierung) an GluN2A- und GluN2BUntereinhieten zu erzielen, und andererseits um Photoantagonismus (Inhibition) an der GluN2A-Untereinheit, oder auch der GluN1-Untereinheit, die allen NMDA-Rezeptoren gemeinsam ist, zu erreichen. Berlin, Szobota et al. haben photoaktivierbare GluN2A-Untereinheiten in organotypischen Hippocampuskulturen exprimiert (Berlin et al, 2016), in welchen diese mit endogenen GluN1-Untereinheiten assemblieren und funktionelle NMDA-Rezeptoren bilden (Abbildung 3). Dabei zeigte sich, dass die lokale Lichtaktivierung der MAG-markierten GluN2A-Untereinheiten ausreichend war, um $\mathrm{Ca}^{2+}$-Signale in einzelnen, dendritischen Dornenfortsätzen zu erzeugen, und dass die Aktivierung dieser postsynaptischen GluN2A-Rezeptoren ausreichend war, um ein Anschwellen der Dorne zu induzieren (Abbildung 3 C, D), was als Hinweis auf eine erfolgte Langzeitpotenzierung gedeutet werden kann. Die Aktivierung von präsynaptischen NMDA-Rezeptoren und anderen GluRs war offenkundig nicht notwendig, um diese Antwort hervorzurufen. Auf ähnliche Wiese hat die Verwendung photoantagonistischer Varianten gezeigt, dass eine partielle Inhibition der GluN1/GluN2A - Untereinheiten ausreichend ist, um ein Anschwellen der Dorne und die Induktion der Langzeitpotenzierung an hippocampalen CA3-CA1 - Synapsen zu unterbinden.

Der Erfolg der MAG-Liganden hat auch das Interesse an alternativen Markierungsstrategien geweckt. So wurde für mGluRs kürzlich ein SNAP-basierter Ansatz implementiert (Broichhagen et al., 2015). In diesem Ansatz wird extrazellulär an den gewünschten Rezeptor eine SNAP-Domäne fusioniert, die mit einem Benzylguanin-Azobenzol-Glutamat- (BGAG) Photoschalter modifiziert werden kann. Das SNAP-Labeling bietet, im Vergleich zu Cysteinmodifikationen, eine höhere Spezifität, erfordert geringere Ligandenkonzentrationen und ist möglicherweise einfacher auf neue Rezeptorsubtypen zu übertragen. Durch die Kombination von SNAP- und CLIP-Tags wird es auch möglich, zwei verschiedene Rezeptorpopulationen im gleichen Präparat zu steuern (Levitz et al., 2017). Alternativ wurde auch eine affinitätsbasierte Kopplung beschrieben, die nicht genetisch kodiert ist und es somit ermöglicht, endogene Rezeptoren zu manipulieren (Izquierdo-Serra et al., 2016).

Chemo-optogenetische Ansätze sind nicht auf die Kontrolle von GluRs beschränkt. Ähnliche photoschaltbare Liganden wurden auch für weitere Rezeptorfamilien entwickelt, einschließlich nikotinischer Acetylcholinrezeptoren (Tochitsky et al., 2012), GABA ${ }_{\mathrm{A}}$-Rezeptoren (Lin et al., 2015; Lin et al., 2018) und Dopaminrezeptoren (Donthamsetti et al., 2017). Auf ähnliche Weise erlauben es photoschaltbare Porenblocker, diverse Kaliumkanaltypen zu kontrollieren (Banghart et al., 2004; Sandoz und Levitz, 2013). Ferner können Azobenzolphotoschalter zur allosterischen Kontrolle von Rezeptoren genutzt werden, z. B. durch die Modifikation der Porenregion in Purin-sensitiven P2X Rezeptoren (Browne et al., 2014; Habermacher 

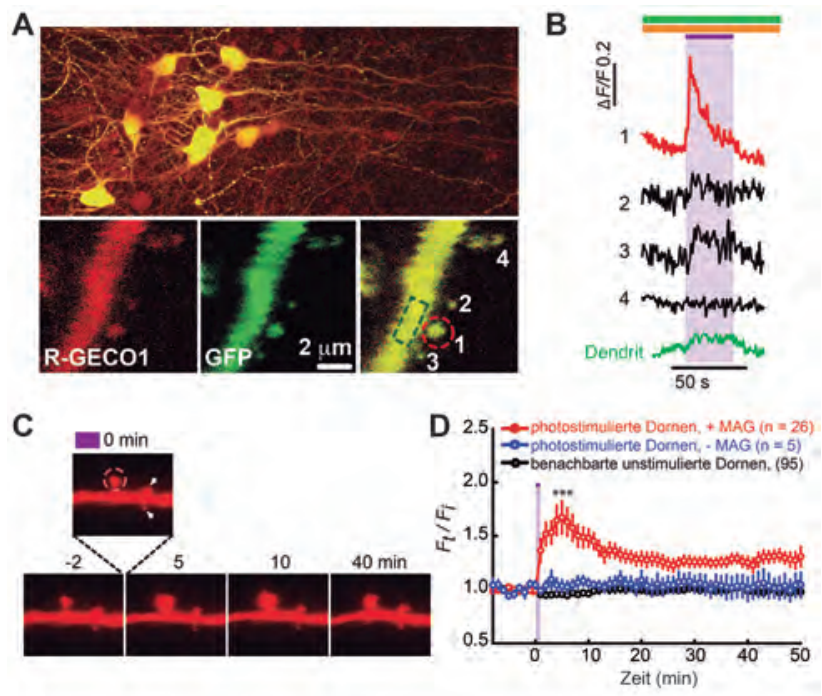

Abb. 3: Die Photoaktivierung postsynaptischer GluN2A NMDARezeptoren führt zu einem Kalziumeinstrom und einem Anschwellen der Dornenfortsätze. A: Organotypischer Hirnschnitt des Hippocampus, in dem CA1-Neurone den rot-fluoreszierenden Calciumsensor R-GECO1 (rot), GFP (grün) und die photoaktivierbare NMDA-Rezeptoruntereinheit GluN2A(V713C) exprimieren. B: Markierung der GluN2A(V713C)-Untereinheiten mit einem MAG-Photoschalter und transiente Photoaktivierung mit $405 \mathrm{~nm}$ Licht führt im stimulierten Dorn (Region 1) zu einem ausgeprägten Kalziumeinstrom (Fluoreszenzzunahme von R-GECO1), aber nicht in den benachbarten Dornen (Region 2-4), und nur zu einem geringen Anstieg im Dendriten. C: Anschwellen eines einzelnen Dorns nach MAG-GluN2A(V713C) Photoaktivierung für 1-2 $\min (t=0)$, sichtbar gemacht durch die Koexpression von rot-fluoreszierendem tdTomato. D: Das Anschwellen der Dorne ist auf die Photoaktivierung zurückzuführen, da dieses nicht in benachbarten Dornen und nicht unter Kontrollbedingungen beobachtet wird. Nach der initialen Expansion bleiben die Dorne für mind. 45 min vergrößert. Die Abbildung wurde modifiziert aus Berlin et al., 2016 entnommen.

et al., 2016), oder, im Falle von NMDA-Rezeptoren, durch den Einbau einer Azobenzolaminosäure mittels Stopcodonsuppression (Klippenstein et al., 2017). Andere nichtnatürliche, photosensitive Aminosäuren wurden auch für die selektive Inaktivierung von Rezeptoren genutzt (Klippenstein et al., 2014).

\section{Weitere Ansätze, um synaptische Signalleitungsproteine mit Licht zu kontrollieren}

Zahlreiche weitere optogenetische Ansätze wurden entwickelt, die für die Manipulation synaptischer Signalleitungsprozesse genutzt werden können. Ein frühes und wichtiges Beispiel ist die Kontrolle von Sekundärbotenstoffen mithilfe des Rhodopsins aus Wirbeltieren, einem GPCR, der durch die Aktivierung von $\mathrm{G}_{\mathrm{i} / \mathrm{o}}$-Proteinen spannungsgesteuerte Kalziumkanäle inhibiert und Kaliumkanäle der GIRK-Familie aktiviert (Li et al., 2005). Rhodopsine wurden zudem genutzt, um ligandengesteuerte GPCRs zu emulieren, indem chimäre Rezeptoren generiert wurden, die die intrazellulären Regionen umfassen, die die Signalleitungs- und Traffickingeigenschaften bestimmen. Dies wurde erstmals mit einer Chimäre aus Rhodopsin und einem $\beta 2$-Adrenozeptor gezeigt (Kim et al., 2005), und später dann unter der Bezeichnung Opto-XRs auch für die Verwendung in Neuronen weiterentwickelt (Airan et al., 2009). Ein ähnliches Vorgehen wurde genutzt, um einen lichtgesteuerten GPCR zu konstruieren, der den Serotoninrezeptor 5-HT $1 \mathrm{a}$ nachbildet (Oh et al., 2010), ein Vorgehen, welches inzwischen auch auf weitere Serotoninrezeptorsubtypen und die Verwendung von Opsinen der Sehzäpfchen sowie Melanopsin ausgeweitet wurde (Masseck et al., 2014; Spoida et al., 2014). Dieser Ansatz wurde kürzlich auch genutzt, um die Signalleitung von „orphan GPCRs“ zu untersuchen, also solchen GPCRs, für die noch kein Ligand identifiziert werden konnte (Morri et al., 2018).

Zahlreiche weitere optogenetische Ansätze nutzen lichtsensitive Proteindomänen, um zelluläre Prozesse zu kontrollieren. Viele dieser Ansätze greifen auf LOVDomänen oder Phytochrome zurück, welche es erlauben, Konformationsänderungen zu induzieren, oder die Assoziation und Lokalisation von Proteinen zu kontrollieren (Möglich und Moffat, 2010). Beispiele dafür sind optogenetische Werkzeuge, die es ermöglichen, die Transkription, das Vesikeltrafficking (van Bergeijk et al., 2015), das Proteinscaffolding (Sinnen et al., 2017), oder die Aggregation (Lee et al., 2014), Inaktivierung und den Abbau von Proteinen zu kontrollieren, die alle auch für die Untersuchung des Rezeptorsignalings genutzt werden könnten.

\section{Ausblick}

Die Kombination von genetischen und optischen Methoden sollte es erlauben, einige der lange bestehenden Fragestellungen anzugehen, die mit dem Studium synaptischer Signalleitungsprozesse verbunden sind. Einige Beispiele: Die gezielte Anregung von Rezeptoren in den verschiedenen synaptischen Kompartimenten kann genutzt werden, um die Interaktion zwischen Neuronen und Gliazellen zu untersuchen. Die hohe Zeitauflösung optischer Methoden sollte es erlauben, die funktionellen 
Konsequenzen verschiedener Aktivierungsmuster herauszuarbeiten, sowohl auf kurzen Zeitskalen, welche z. B. für die Spikezeit-abhängige Plastizität bedeutsam sind, als auch auf langen Zeitskalen, die für Entwicklungsprozesse von Bedeutung sind. Die Möglichkeit verschiedene Rezeptorsubtypen getrennt voneinander ansprechen zu können, ermöglicht es, deren individuelle Funktion, aber auch deren Zusammenspiel zu untersuchen (Reiner und Levitz, 2018). Die Manipulation von Rezeptoren in ausgewählten Zellpopulationen im lebenden Tier sollte es schließlich ermöglichen, genauer zu definieren, welche Rezeptorsubtypen und Hirnareale für therapeutische Interventionen in Betracht gezogen werden sollten. Die Entwicklung und Nutzung dieser Techniken dürfte uns somit spannende, neue Einblicke liefern - von einzelnen Synapsen, über die Funktion von Netzwerken, bis hin zu Kognition und Verhalten.

\section{Glossar}

5-HT
AMA-Rezeptor
AMPA-Rezeptoren

Azobenzol

BGAG-Photoschalter

$\mathrm{GABA}_{\mathrm{A}}-$ Rezeptor

GIRK-Kanäle

GluK2

GluNx

GPCR

iGluR

LOV-Domänen

MAG-Photoschalter

mGluR
Serotonin (5-Hydroxytryptamin)-Rezeptor $1 \mathrm{~A}\left(\mathrm{G}_{\mathrm{i}}\right.$-gekoppelter GPCR)

iGluR-Unterfamilie, benannt nach dem Agonisten $\alpha$-Amino-3-hydroxy-5-methyl-4isoxazolpropionsäure

eine chemische Gruppe, die einer lichtinduzierten trans-cis bzw. cis-trans-Isomerisierung unterliegt und häufig als Photoschalter Verwendung findet

Benzylguanin-Azzobenzol-GlutamatPhotoschalter für die optische Kontrolle von iGluRs (ermöglicht die Anknüpfung an SNAP-Tags)

$\mathrm{Y}$-Aminobuttersäurerezeptor A (ligandengesteuerter Chloridkanal)

$G$ protein-coupled inwardly-rectifying potassium channels (G-Protein-gekoppelte einwärtsgleichrichtende Kaliumkanäle) Untereinheit 2 der Kainatrezeptorfamilie (ehemals iGluR6)

Untereinheiten der NMDA-Rezeptorfamilie G-Protein-gekoppelter Rezeptor ionotroper Glutamatrezeptor (ligandengesteuerter lonenkanal)

light-oxygen-voltage-sensing Domänen, eine Familie flavinhaltiger Proteindomänen, die bei Bestrahlung ihre Konformation ändern

Maleimid-Azobenzol-Glutamat-Photoschalter für die optische Kontrolle von GluRs (ermöglicht die Anknüpfung an Cysteinreste)

metabotroper Glutamatrezeptor (GPCR)
NMDA-Rezeptoren

P2X-Rezeptoren

SNAP/CLIP-Tags

TRP-Kanäle
iGluR-Unterfamilie, benannt nach dem Agonisten N-Methyl-D-Aspartat Bezeichnung für ionotrope, purinerge Rezeptoren (ATP-gesteuerte Kationenkanäle)

Proteintags, die mit $O^{6}$-Benzylguaninderivaten (SNAP) bzw. $\mathrm{O}^{2}$-Benzylcytosinderivaten (CLIP) reagieren, und daher zur kovalenten Anknüpfung entsprechender Farbstoffe und Photoschalter genutzt werden können transient receptor potential channels, eine Klasse von Kationenkanälen, die u. a. durch Liganden aktiviert werden können

\section{Literatur}

Airan, R.D., Thompson, K.R., Fenno, L.E., Bernstein, H. and Deisseroth, K. (2009). Temporally precise in vivo control of intracellular signalling. Nature 458, 1025-1029.

Banghart, M., Borges, K., Isacoff, E., Trauner, D. and Kramer, R.H. (2004). Light-activated ion channels for remote control of neuronal firing. Nat. Neurosci. 7, 1381-1386.

Barber, D.M., Liu, S.A., Gottschling, K., Sumser, M., Hollmann, M. and Trauner, D. (2017). Optical control of AMPA receptors using a photoswitchable quinoxaline-2,3-dione antagonist. Chem. Sci. 8, 611-615.

Berlin, S., Szobota, S., Reiner, A., Carroll, E.C., Kienzler, M.A., Guyon, A., Xiao, T., Trauner, D. and Isacoff, E.Y. (2016). A family of photoswitchable NMDA receptors. eLife 5, e12040.

Boyden, E.S., Zhang, F., Bamberg, E., Nagel, G. and Deisseroth, K. (2005). Millisecond-timescale, genetically targeted optical control of neural activity. Nat. Neurosci. 8, 1263-1268.

Broichhagen, J., Damijonaitis, A., Levitz, J., Sokol, K.R., Leippe, P., Konrad, D., Isacoff, E.Y. and Trauner, D. (2015). Orthogonal optical control of a $\mathrm{G}$ protein-coupled receptor with a SNAP-tethered photochromic ligand. ACS Cent. Sci. 1, 383-393.

Browne, L.E., Nunes, J.P., Sim, J.A., Chudasama, V., Bragg, L., Caddick, S. and North, R.A. (2014). Optical control of trimeric $\mathrm{P} 2 \mathrm{X}$ receptors and acid-sensing ion channels. Proc. Natl. Acad. Sci. USA 111, 521-526.

Carroll, E.C., Berlin, S., Levitz, J., Kienzler, M.A., Yuan, Z., Madsen, D., Larsen, D.S. and Isacoff, E.Y. (2015). Two-photon brightness of azobenzene photoswitches designed for glutamate receptor optogenetics. Proc. Natl. Acad. Sci. USA 112, E776-785.

Deisseroth, K., and Hegemann, P. (2017). The form and function of channelrhodopsin. Science 357, eaan5544.

Donthamsetti, P.C., Winter, N., Schönberger, M., Levitz, J., Stanley, C., Javitch, J.A., Isacoff, E.Y. and Trauner, D. (2017). Optical control of dopamine receptors using a photoswitchable tethered inverse agonist. J. Am. Chem. Soc. 139, 18522-18535.

Ellis-Davies, G.C. (2007). Caged compounds: photorelease technology for control of cellular chemistry and physiology. Nat. Methods 4, 619-628.

Fehrentz, T., Schönberger, M. and Trauner, D. (2011). Optochemical genetics. Angew. Chem. Int. Ed. Engl. 50, 12156-12182.

Gaub, B.M., Berry, M.H., Holt, A.E., Reiner, A., Kienzler, M.A., Dolgova, N., Nikonov, S., Aguirre, G.D., Beltran, W.A., Flannery, 
J.G. and Isacoff, E.Y. (2014). Restoration of visual function by expression of a light-gated mammalian ion channel in retinal ganglion cells or ON-bipolar cells. Proc. Natl. Acad. Sci. USA 111, E5574-5583.

Gorostiza, P., Volgraf, M., Numano, R., Szobota, S., Trauner, D. and Isacoff, E.Y. (2007). Mechanisms of photoswitch conjugation and light activation of an ionotropic glutamate receptor. Proc. Natl. Acad. Sci. USA 104, 10865-10870.

Habermacher, C., Martz, A., Calimet, N., Lemoine, D., Peverini, L., Specht, A., Cecchini, M. and Grutter, T. (2016).

Photo-switchable tweezers illuminate pore-opening motions of an ATP-gated P2X ion channel. eLife 5, e11050.

Hollmann, M. and Heinemann, S. (1994). Cloned glutamate receptors. Annu. Rev. Neurosci. 17, 31-108.

Jacobi, E. and von Engelhardt, J. (2017). Diversity in AMPA receptor complexes in the brain. Curr. Opin. Neurobiol. 45, 32-38.

Izquierdo-Serra, M., Bautista-Barrufet, A., Trapero, A., GarridoCharles, A., Díaz-Tahoces, A., Camarero, N., Pittolo, S., Valbuena, S., Pérez-Jiménez, A., Gay, M. et al. (2016). Optical control of endogenous receptors and cellular excitability using targeted covalent photoswitches. Nat. Commun. 7, 12221.

Kauwe, G. and Isacoff, E.Y. (2013). Rapid feedback regulation of synaptic efficacy during high-frequency activity at the Drosophila larval neuromuscular junction. Proc. Natl. Acad. Sci. USA 110, 9142-9147.

Kienzler, M.A., Reiner, A., Trautman, E., Yoo, S., Trauner, D. and Isacoff, E.Y. (2013). A red-shifted, fast-relaxing azobenzene photoswitch for visible light control of an ionotropic glutamate receptor. J. Am. Chem. Soc. 135, 17683-17686.

Kim, J.M., Hwa, J., Garriga, P., Reeves, P.J., RajBhandary, U.L. and Khorana, H.G. (2005). Light-driven activation of beta 2-adrenergic receptor signaling by a chimeric rhodopsin containing the beta 2-adrenergic receptor cytoplasmic loops. Biochemistry 44, 2284-2292.

Kim, C.K., Adhikari, A. and Deisseroth, K. (2017). Integration of optogenetics with complementary methodologies in systems neuroscience. Nat. Rev. Neurosci. 18, 222-235.

Klippenstein, V., Ghisi, V., Wietstruk, M. and Plested, A.J. (2014). Photoinactivation of glutamate receptors by genetically encoded unnatural amino acids. J. Neurosci. 34, 980-991.

Klippenstein, V., Hoppmann, C., Ye, S., Wang, L. and Paoletti, P. (2017). Optocontrol of glutamate receptor activity by single side-chain photoisomerization. eLife 6, e25808.

Kramer, R.H., Mourot, A. and Adesnik, H. (2013). Optogenetic pharmacology for control of native neuronal signaling proteins. Nat. Neurosci. 16, 816-823.

Laprell, L., Repak, E., Franckevicius, V., Hartrampf, F., Terhag, J., Hollmann, M., Sumser, M., Rebola, N., DiGregorio, D.A. and Trauner, D. (2015). Optical control of NMDA receptors with a diffusible photoswitch. Nat. Commun. 6, 8076.

Lee, S., Park, H., Kyung, T., Kim, N.Y., Kim, S., Kim, J. and Heo, W.D. (2014). Reversible protein inactivation by optogenetic trapping in cells. Nat. Methods 11, 633-636.

Levitz, J., Pantoja, C., Gaub, B., Janovjak, H., Reiner, A., Hoagland, A., Schoppik, D., Kane, B., Stawski, P., Schier, A.F. et al. (2013). Optical control of metabotropic glutamate receptors. Nat. Neurosci. 16, 507-516.

Levitz, J., Habrian, C., Bharill, S., Fu, Z., Vafabakhsh, R. and Isacoff, E.Y. (2016a). Mechanism of assembly and cooperativity of homomeric and heteromeric metabotropic glutamate receptors. Neuron 92, 143-159.

Levitz, J., Popescu, A.T., Reiner, A. and Isacoff, E.Y. (2016b). A toolkit for orthogonal and in vivo optical manipulation of ionotropic glutamate receptors. Front. Mol. Neurosci. 9, 2.

Levitz, J., Broichhagen, J., Leippe, P., Konrad, D., Trauner, D. and Isacoff, E.Y. (2017). Dual optical control and mechanistic insights into photoswitchable group II and III metabotropic glutamate receptors. Proc. Natl. Acad. Sci. USA 114, E3546-3554.

Li, X., Gutierrez, D.V., Hanson, M.G., Han, J., Mark, M.D., Chiel, H., Hegemann, P., Landmesser, L.T. and Herlitze, S. (2005). Fast noninvasive activation and inhibition of neural and network activity by vertebrate rhodopsin and green algae channelrhodopsin. Proc. Natl. Acad. Sci. USA 102, 17816-17821.

Li, D., Hérault, K., Isacoff, E.Y., Oheim, M. and Ropert, N. (2012). Optogenetic activation of LiGluR-expressing astrocytes evokes anion channel-mediated glutamate release. J. Physiol. 590, 855-873.

Lin, W.C., Tsai, M.C., Davenport, C.M., Smith, C.M., Veit, J., Wilson, N.M., Adesnik, H. and Kramer, R.H. (2015). A Comprehensive optogenetic pharmacology toolkit for in vivo control of GABA(A) receptors and synaptic inhibition. Neuron 88, 879-891.

Lin, W.C., Tsai, M.C., Rajappa, R. and Kramer, R.H. (2018). Design of a highly bistable photoswitchable tethered ligand for rapid and sustained manipulation of neurotransmission. J. Am. Chem. Soc. 140, 7445-7448.

Luo, L., Callaway, E.M. and Svoboda, K. (2018). Genetic dissection of neural circuits: A decade of progress. Neuron 98, 256-281.

Masseck, O.A., Spoida, K., Dalkara, D., Maejima, T., Rubelowski, J.M., Wallhorn, L., Deneris, E.S. and Herlitze, S. (2014). Vertebrate cone opsins enable sustained and highly sensitive rapid control of Gi/o signaling in anxiety circuitry. Neuron 81 , 1263-1273.

Monyer, H., Seeburg, P.H. and Wisden, W. (1991). Glutamateoperated channels: developmentally early and mature forms arise by alternative splicing. Neuron 6, 799-810.

Morri, M., Sanchez-Romero, I., Tichy, A.M., Kainrath, S., Gerrard, E.J., Hirschfeld, P.P., Schwarz, J. and Janovjak, H. (2018). Optical functionalization of human Class A orphan G-protein-coupled receptors. Nat. Commun. 9, 1950.

Möglich, A. and Moffat, K. (2010). Engineered photoreceptors as novel optogenetic tools. Photochem. Photobiol. Sci. 9, 1286-1300.

Nagel, G., Ollig, D., Fuhrmann, M., Kateriya, S., Musti, A.M., Bamberg, E. and Hegemann, P. (2002). Channelrhodopsin-1: a light-gated proton channel in green algae. Science 296, 2395-2398.

Nagel, G., Brauner, M., Liewald, J.F., Adeishvili, N., Bamberg, E. and Gottschalk, A. (2005). Light activation of channelrhodopsin-2 in excitable cells of Caenorhabditis elegans triggers rapid behavioral responses. Curr. Biol. 15, 2279-2284.

Oh, E., Maejima, T., Liu, C., Deneris, E. and Herlitze, S. (2010). Substitution of 5-HT1A receptor signaling by a light-activated G protein-coupled receptor. J. Biol. Chem. 285, 30825-30836.

Pittolo, S., Gómez-Santacana, X., Eckelt, K., Rovira, X., Dalton, J., Goudet, C., Pin, J.P., Llobet, A., Giraldo, J., Llebaria, A. and Gorostiza, P. (2014). An allosteric modulator to control endogenous $G$ protein-coupled receptors with light. Nat. Chem. Biol. 10, 813-815. 
Reiner, A. and Isacoff, E.Y. (2013). The Brain Prize 2013: The optogenetics revolution. Trends Neurosci. 36, 557-560.

Reiner, A. and Isacoff, E.Y. (2014a). Tethered ligands reveal glutamate receptor desensitization depends on subunit occupancy. Nat. Chem. Biol. 10, 273-280.

Reiner, A. and Isacoff, E.Y. (2014b). Photoswitching of cell surface receptors using tethered ligands. Methods Mol. Biol. 1148, 45-68.

Reiner, A., Levitz, J. and Isacoff, E.Y. (2015). Controlling ionotropic and metabotropic glutamate receptors with light: principles and potential. Curr. Opin. Pharmacol. 20, 135-143.

Reiner, A. and Levitz, J. (2018). Glutamatergic signaling in the central nervous system: Ionotropic and metabotropic receptors in concert. Neuron 98, 1080-1089.

Rullo, A., Reiner, A., Reiter, A., Trauner, D., Isacoff, E.Y. and Woolley, G.A. (2014). Long wavelength optical control of glutamate receptor ion channels using a tetra-ortho-substituted azobenzene derivative. Chem. Commun. 50, 14613-14615.

Sandoz, G. and Levitz, J. (2013). Optogenetic techniques for the study of native potassium channels. Front. Mol. Neurosci. 6, 6 .

Schwenk, J., Harmel, N., Brechet, A., Zolles, G., Berkefeld, H., M.ller, C.S., Bildl, W., Baehrens, D., H,ber, B., Kulik, A. et al. (2012). High-resolution proteomics unravel architecture and molecular diversity of native AMPA receptor complexes. Neuron 74, 621-633.

Sinnen, B.L., Bowen, A.B., Forte, J.S., Hiester, B.G., Crosby, K.C., Gibson, E.S., Dell'Acqua, M.L. and Kennedy, M.J. (2017). Optogenetic control of synaptic composition and function. Neuron 93, 646-660.

Spoida, K., Masseck, O.A., Deneris, E.S. and Herlitze, S. (2014). $\mathrm{Gq} / 5-\mathrm{HT} 2 \mathrm{c}$ receptor signals activate a local GABAergic inhibitory feedback circuit to modulate serotonergic firing and anxiety in mice. Proc. Natl. Acad. Sci. USA 111, 6479-6484.

Szobota, S., Gorostiza, P., Del Bene, F., Wyart, C., Fortin, D.L., Kolstad, K.D., Tulyathan, O., Volgraf, M., Numano, R., Aaron, H.L. et al. (2007). Remote control of neuronal activity with a light-gated glutamate receptor. Neuron 54, 535-545.

Tochitsky, I., Banghart, M.R., Mourot, A., Yao, J.Z., Gaub, B., Kramer, R.H. and Trauner, D. (2012). Optochemical control of genetically engineered neuronal nicotinic acetylcholine receptors. Nat. Chem. 4, 105-111.

Traynelis, S.F., Wollmuth, L.P., McBain, C.J., Menniti, F.S., Vance, K.M., Ogden, K.K., Hansen, K.B., Yuan, H., Myers, S.J. and Dingledine, R. (2010). Glutamate receptor ion channels: structure, regulation, and function. Pharmacol. Rev. 62, 405-496.

van Bergeijk, P., Adrian, M., Hoogenraad, C.C. and Kapitein, L.C. (2015). Optogenetic control of organelle transport and positioning. Nature 518, 111-114.

Volgraf, M., Gorostiza, P., Numano, R., Kramer, R.H., Isacoff, E.Y. and Trauner, D. (2006). Allosteric control of an ionotropic glutamate receptor with an optical switch. Nat. Chem. Biol. 2, 47-52.

Wietek, J., Rodriguez-Rozada, S., Tutas, J., Tenedini, F., Grimm, C., Oertner, T.G., Soba, P., Hegemann, P. and Wiegert, J.S. (2017). Anion-conducting channelrhodopsins with tuned spectra and modified kinetics engineered for optogenetic manipulation of behavior. Sci. Rep. 7, 14957.

Wiegert, J.S., Mahn, M., Prigge, M., Printz, Y. and Yizhar, O. (2017). Silencing neurons: Tools, applications, and experimental constraints. Neuron 95, 504-529.
Wyart, C., Del Bene, F., Warp, E., Scott, E.K., Trauner, D., Baier, H. and Isacoff, E.Y. (2009). Optogenetic dissection of a behavioural module in the vertebrate spinal cord. Nature 461, 407-410.

Zemelman, B.V. and Miesenböck, G. (2001). Genetic schemes and schemata in neurophysiology. Curr. Opin. Neurobiol.11, 409-414.

Zemelman, B.V., Nesnas, N., Lee, G.A. and Miesenböck, G. (2003). Photochemical gating of heterologous ion channels: remote control over genetically designated populations of neurons. Proc. Natl. Acad. Sci. USA 100, 1352-1357.

Anmerkung: Die englische Version des Artikels ist online verfügbar unter https://doi.org/10.1515/nf-2018-A014

\section{Autoreninformationen}

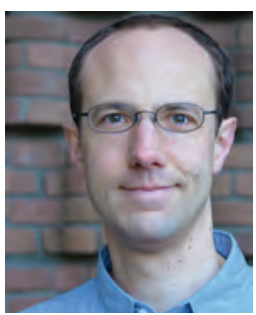

Andreas Reiner

Fakultät für Biologie und Biotechnologie, Ruhr-Universität Bochum

Universitätsstraße 150

44801 Bochum

E-Mail: andreas.reiner@rub.de

Andreas Reiner ist Juniorprofessor und Leiter der Nachwuchsgruppe Zelluläre Neurobiologie an der Ruhr-Universität Bochum. Er hat Biochemie an der Universität Bayreuth studiert und bei Thomas Kiefhaber am Biozentrum der Universität Basel promoviert. Nach einem Forschungsaufenthalt an der Technischen Universität München hat er als Postdoc im Labor von Ehud Y. Isacoff an der University of California Berkeley begonnen, neuronale Glutamatrezeptoren mit opto-chemischen Methoden zu untersuchen. 2015 wurde er mit einem NRW-Rückkehrerstipendium ausgezeichnet. Er ist insbesondere an der Umsetzung neuer optischer Techniken interessiert, um neue mechanistische und physiologische Aspekte der Funktion von Glutamatrezeptoren zu untersuchen. 